\title{
MMW-NOTFALLCHECKLISTE
}

\section{Plötzlicher Schmerz im After}

\author{
H. S. FÜESSL
}

\author{
Auf einer längeren Bahnreise verspürt ein 42-jähriger Mann \\ plötzlich Schmerzen im After. Er sucht die Toilette auf und \\ ertastet in der Aftergegend einen etwa kirschgroßen Knoten, \\ der sehr druckschmerzhaft ist. Dieser Tastbefund beunruhigt \\ ihn sehr, sodass er am nächsten Vormittag eine proktologische \\ Fachpraxis aufsucht.
}

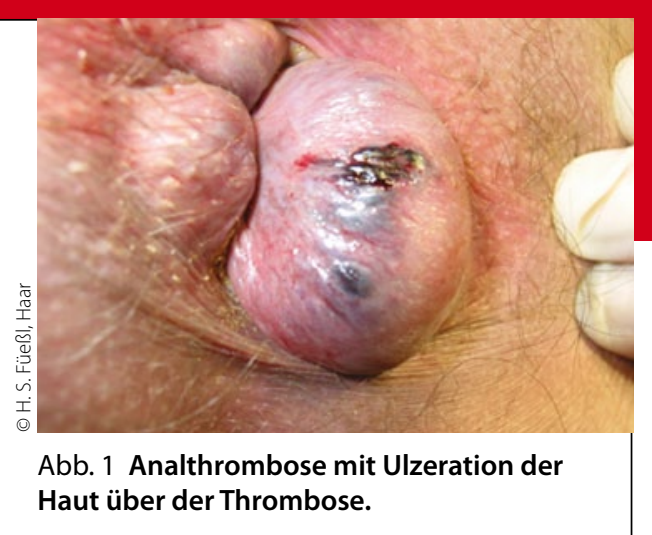

- Anamnestisch berichtet der Mann über gelegentliche, v. a. bei hartem Stuhl auftretende Blutauflagerungen auf dem Toilettenpapier. Diesen Befund interpretierte er als Hämorrhoiden, für die er keine Behandlungsnotwendigkeit sah. Bei der Untersuchung stellt sich der in Abb. 1 gezeigte Befund dar.

\section{Diagnostik}

- Die Diagnose der Analthrombose wird anhand des typischen Aspekts gestellt. - Bei der Inspektion und der digitalen Untersuchung finden sich am Analrand oder subanodermal im Analkanal prall elastische bis derbe, bläulich rote Knoten, deren Größe von Glasstecknadelkopf- bis Pflaumengröße reichen kann.

- Manchmal finden sich auch mehrere kleine Thrombosen wie an einer Perlschnur angeordnet.

- Die Palpation ist im frühen Stadium in der Regel druckschmerzhaft. Frische Thrombosen bluten in der Regel nicht.

- Durch ein Begleitödem kann die Sicht auf die eigentliche Thrombose beeinträchtigt sein.

- Differenzialdiagnostisch muss man an Abszesse, thrombosierte oder inkarzerierte Hämorrhoiden, Marisken, einen Analprolaps, Analfibrome, Melanome und ein Analkarzinom denken.

\section{Definition}

Bei der Analthrombose handelt es sich um Koagel, die aus den subkutan verlaufenden Venen des Plexus hemorrhoidalis caudalis stammen. Die Analthrombose unterscheidet sich vom Hämorrhoidalleiden, bei dem eine Erweiterung des arteriell gespeisten Plexus hemorrhoidalis vorliegt. Zwischen beiden Erkrankungen besteht kein ursächlicher Zusammenhang. Sie werden häufig verwechselt.

Synonyma sind Perianalthrombose, perianale Thrombose und Analvenenthrombose. Der im Amerikanischen übliche Ausdruck „anal hematoma“ widerspricht den anatomischen Gegebenheiten völlig, da es sich nicht um ein extravasales Hämatom, sondern um eine Thrombose in einer Vene handelt. Auch die hierzulande häufige Bezeichnung „äußere Hämorrhoiden“ ist anatomisch nicht korrekt.

\section{Ätiopathogenese}

Die Ätiologie der Analthrombose, die nahezu alle Altersgruppen betrifft, ist weitgehend unklar. Als Auslöser werden die Pressbelastung durch harten Stuhl, aber auch Durchfälle, Schwangerschaft, übermäßiger Alkoholgenuss, Analverkehr oder der Genuss von scharfen Speisen postuliert. Trotz der anatomischen Unterschiede zum Hämorrhoidalleiden scheinen für das Entstehen von Analthrombosen vergrößerte Hämorrhoidalpolster doch eine Rolle zu spielen.

\section{Verlauf}

Abhängig von der initialen Größe der Thrombose ist eine spontane Rückbildung der Schmerzen und eine vollständige Resorption des Thrombus innerhalb weniger Wochen möglich. Unter Umständen kommt es infolge einer Drucknekrose zur Hautulzeration (Abb. 1) über der Thrombose mit nachfolgender Blutung und evtl. spontanem Abgang des Gerinnungsthrombus. Dies lässt die Beschwerden gelegentlich schlagartig verschwinden.

\section{Therapie}

- Zunächst konservatives Vorgehen, da sich die Beschwerden häufig spontan zurückbilden. Dabei werden lokal und/ oder systemisch antiphlogistische und analgetische Maßnahmen eingesetzt.

- Bei großen Befunden mit erheblichen Beschwerden sollte man eine komplette Entfernung des Knotens nach vorheriger Lokalanästhesie ohne Naht durchführen, da eine Inzision oder eine Thrombusexpression mit einer erheblichen Rezidivrate einhergeht.

- Je später sich der Patient nach dem Ereignis vorstellt, umso seltener wird man die Indikation zur Operation stellen.

\section{Anschrift des Verfassers:}

Prof. Dr. med. Hermann S. FüeßI

Leiter Somatischer Querschnittsbereich, IsarAmper-Klinikum, Klinikum München-Ost

Ringstr. 33a, D-85540 Haar

E-Mail: hermann.fueessl@iak-kmo.de

\section{Kasuistik - Wie ging es weiter?}

Nach entsprechender Aufklärung wünschte der Patient keine Operation, sondern wollte den Spontanverlauf abwarten. Tatsächlich bildeten sich die Beschwerden innerhalb einer Woche weitgehend zurück. 